\title{
REVIEW ARTICLE \\ Mycophenolate Mofetil versus Cyclophosphamide for Induction Treatment of Lupus Nephritis
}

\author{
N Mahmood ${ }^{1}$, F Mahmood $^{2}$, SI Shumi ${ }^{3}$, MMR Siddiqui $^{4}$
}

\begin{abstract}
Recent studies have suggested that mycophenolate mofetil (MMF) may offer advantages over intravenous cyclophosphamide (IVC) for the treatment of lupus nephritis, but these therapies have not been compared in an international randomized, controlled trial. Here, the comparison of MMF and IVC as induction treatment for active lupus nephritis in a multinational, two-phase (induction and maintenance) study was shown in the different study. Lupus nephritis (LN) occurs in up to $60 \%$ of adults with systemic lupus erythematosus (SLE) and predicts poor survival. The prevalence of SLE and LN and treatment response vary by age, gender, location, and race/ethnicity; LN is especially common in black and Hispanic patients in the United States. MMF was at least as effective as IVC in induction treatment in previous trials in Hong Kong, Malaysia, China, and the United States. Meta-analyses of these and smaller trials suggested that MMF may offer advantages over IVC, but they have not yet been compared in an international randomized, controlled trial. Many comparative studies were undertaken in patients with LN, a two-part trial to assess the efficacy and safety of MMF as induction therapy and subsequently as maintenance therapy for LN. This article will describe the comparison of MMF with IVC, both with corticosteroids.
\end{abstract}

\section{Introduction}

Recent studies have suggested that mycophenolate mofetil (MMF) may offer advantages over intravenous cyclophosphamide (IVC) for the treatment of lupus nephritis, but these therapies have not been compared in an international randomized, controlled trial.

Lupus nephritis (LN) occurs in up to $60 \%$ of adults with systemic lupus erythematosus (SLE) and predicts poor survival. ${ }^{1,2}$ The prevalence of SLE and LN and treatment response vary by age, gender, location, and race/ethnicity; $\mathrm{LN}$ is especially common in black and Hispanic patients in the United States. 3,4

Use of intravenous cyclophosphamide (IVC) is based on studies in the 1970s and 1980s at the
National Institutes of Health (NIH). ${ }^{5,6}$ The subsequent induction regimen, widely considered the standard of care, requires monthly intravenous drug infusions. ${ }^{7}$ Response is often slow, and treatment fails to control LN fully and is associated with increased risks for adverse effects, including gonadal toxicity. ${ }^{8,9}$ Among other immunosuppressants, recent studies have focused on mycophenolate mofetil (MMF). ${ }^{10}$ Unlike IVC, MMF has not been associated with an increased risk of bladder or ovarian toxicity in LN or during long-term use after transplantation. ${ }^{10,11}$

MMF was at least as effective as IVC in induction treatment in previous trials in Hong Kong, ${ }^{12,13}$ Malaysia, ${ }^{14}$ China, and the United States. ${ }^{12-16}$ Meta-

IDr. Nazneen Mahmood, Associate Professor and Head, Department of Nephrology, Anwer Khan Modern Medical College \& Hospital

2Dr. Farhana Mahmood, Assistant Professor, Department of Medicine, Chattagram Maa O Shishu Hospital Medical College, Agrabad, Chittagong, Bangladesh

${ }^{3}$ Dr. Shamima Islam Shumi, Biochemist, Ansar and VDP Hospital, Shafipur, Gazipur

${ }^{4}$ Dr. Md. Mahmudur Rahman Siddiqui, Associate Professor, Department of Medicine, Anwer Khan Modern Medical College \& Hospital

*Corresponding author

Date of submission: 15. 10.2017, Date of acceptance: 09.11.2017

AKMMC J 2018; 9(1) : 63-67 
analyses of these and smaller trials suggested that MMF may offer advantages over IVC, but they have not yet been compared in an international randomized, controlled trial. ${ }^{17-19}$ GB Appel et al, therefore, undertook one of the largest studies to date in patients with $\mathrm{LN}$, a two-part trial to assess the efficacy and safety of MMF as induction therapy and subsequently as maintenance therapy for LN. This report describes the comparison of MMF with IVC, both with corticosteroids, for the induction treatment of active classes III, IV, and V LN. The hypothesis was that more patients with $\mathrm{LN}$ would respond to MMF than to IVC during 24 wks.

\section{Discussion}

GB Appel et al 2009, report the comparison of MMF and IVC as induction treatment for active lupus nephritis in a multinational, two-phase (induction and maintenance) study. They randomly assigned 370 patients with classes III through V lupus nephritis to open-label MMF (target dosage 3 $\mathrm{g} / \mathrm{d}$ ) or IVC (0.5 to $1.0 \mathrm{~g} / \mathrm{m}^{2}$ in monthly pulses) in a 24-wk induction study. Both groups received prednisone, tapered from a maximum starting dosage of $60 \mathrm{mg} / \mathrm{d}$. The primary end point was a pre-specified decrease in urine protein/creatinine ratio and stabilization or improvement in serum creatinine. Secondary end points included complete renal remission, systemic disease activity and damage, and safety. Overall, they did not detect a significantly different response rate between the two groups: $104(56.2 \%)$ of 185 patients responded to MMF compared with 98 (53.0\%) of 185 to IVC. Secondary end points were also similar between treatment groups. There were nine deaths in the MMF group and five in the IVC group. They did not detect significant differences between the MMF and IVC groups with regard to rates of adverse events, serious adverse events, or infections. Although most patients in both treatment groups experienced clinical improvement, the study did not meet its primary objective of showing that MMF was superior to IVC as induction treatment for lupus nephritis. To determine whether mycophenolate mofetil induces remission of active lupus nephritis as effectively as cyclophosphamide, and to compare adverse effects of these agents ${ }^{20}$.
In another study of C Burchardi and D Schlondarff ,Starting in December 1999, patients with systemic lupus erythematosus, biopsy-proven lupus nephritis (class III-V) and evidence of active disease (e.g. elevated serum creatinine concentration, proteinuria or microscopic hematuria), were prospectively recruited in this open-label, multicenter US study. Exclusion criteria included creatinine clearance $<30 \mathrm{ml} / \mathrm{min}$, serum creatinine $>3.0 \mathrm{mg} / \mathrm{dl}(>265.2$ $\mu \mathrm{mol} / \mathrm{l})$, and previous exposure to mycophenolate mofetil. In addition to prednisone $1 \mathrm{mg} / \mathrm{kg} / \mathrm{day}$, patients were randomized to receive oral mycophenolate mofetil (starting at $1,000 \mathrm{mg} /$ day and increasing to $3,000 \mathrm{mg} /$ day, provided the white blood cell count remained $\geqslant 3,000 / \mathrm{mm} 3$ ) or monthly intravenous cyclophosphamide. Prednisone dosage was reduced by $10-20 \%$ every week or fortnight, depending on disease response. Patients who showed no response after 12 weeks were permitted to cross over to the other therapy. Outcomes at 24 weeks were analyzed by intention to treat.

Complete remission of disease (denoted by $\leqslant 10 \%$ variation from normal levels of serum creatinine, urine protein and urine sediment) was the primary endpoint and partial remission (50\% improvement in abnormal serum creatinine, proteinuria and urine sediment values) was a secondary endpoint.

Of the 140 patients enrolled, 71 were randomized to mycophenolate (14\% male; mean age 32.5 years) and 69 to cyclophosphamide $(6 \%$ male; mean age 31.0 years). After 12 weeks' treatment, responses were noted in 56 and 42 patients, respectively. At 24 weeks, $22.5 \%$ (16) of mycophenolate patients showed complete remission, compared with 5.8\% (4) of cyclophosphamide patients. The absolute difference in complete remission rates between treatments was $16.7 \%$ (95\% CI 5.6-27.9\%; $\mathrm{P}=$ 0.005 ), with the positive value of the lower limit of the $95 \%$ CI indicating that mycophenolate was superior to cyclophosphamide. Rates of partial remission were similar between patients given mycophenolate and those who received cyclophosphamide (29.6\% vs $24.6 \%$; $\mathrm{P}=0.51)$. The overall (complete plus partial) remission rate was higher in the mycophenolate group than in the cyclophosphamide group $(52.1 \%$ vs $30.4 \%$; $\mathrm{P}=$ $0.009)$. Treatment failure occurred less frequently in 
the mycophenolate group than in the cyclophosphamide group $(47.9 \%$ vs $69.6 \%$; $\mathrm{P}=$ 0.01 ). The mycophenolate group had fewer deaths and severe infections than the cyclophosphamide group ( 0 vs 2 and 1 vs 6 , respectively), but more cases of diarrhea (15 vs 2$)^{21}$.

In order to reduce the toxicity of the therapeutic agents used in patients with proliferative lupus nephritis, dose regimens are divided into a 6-month induction phase followed by a maintenance phase, analogous to the approach employed in oncology. Previously, optimal long-term results were obtained using monthly intravenous cyclophosphamide and oral steroids for induction, and intravenous cyclophosphamide (every 3 months) or oral azathioprine for maintenance. In the search for less toxic but equally effective regimens, various drug dosages and combinations have been evaluated. In the Euro-Lupus Nephritis Trial, induction with a reduced dose of cyclophosphamide (six fortnightly doses of $500 \mathrm{mg}$ ) was as effective as a regimen comprising six monthly and two quarterly pulses of high-dose cyclophosphamide with respect to treatment failure, renal remission and renal flare at a median follow-up of 73 months. ${ }^{22}$ Azathioprine was used as maintenance. Subsequently, Contreras et al. found that daily mycophenolate mofetil was less toxic and more effective in maintaining remission over 72 months than quarterly cyclophosphamide, and at least as effective as azathioprine, though the number of patients with long-term data who were analyzed was very small. ${ }^{23}$

Ginzler et al. now question intravenous cyclophosphamide as the gold standard for induction therapy of severe lupus nephritis. In Chinese patients, Chan et al. have previously reported comparable efficacy (partial and complete renal remission) for mycophenolate and daily oral cyclophosphamide induction, with less toxicity for mycophenolate. ${ }^{24}$ In US patients with proliferative lupus nephritis, Ginzler and colleagues now show comparable, if not superior, results for oral mycophenolate compared with intravenous cyclophosphamide for induction. The question therefore arises: why not use mycophenolate, combined with steroids, for both induction and maintenance treatment of severe lupus nephritis?
The Chan et al. and Ginzler et al. reports are in favor of this approach. Not only were initial and final renal outcomes comparable in both studies (after median follow-up periods of 63 months and 36 months respectively), but there were also significantly fewer serious adverse effects in the mycophenolate groups. Because of this, and because of the lack of ovarian dysfunction observed with mycophenolate, the predominantly female lupus nephritis population might be more willing to adopt mycophenolate than cyclophosphamide.

There are, however, some reservations about using mycophenolate as an alternative to cyclophosphamide for induction treatment of severe lupus nephritis, based on the results of the Ginzler et al. study. These include the considerable drop-out rate $(50 \%)$, and the dosage of mycophenolate ( $3 \mathrm{~g} / \mathrm{day})$, which is higher than would be used for a non-AfricanAmerican population. The lack of long-term (i.e. 10-year) efficacy data for many patients on mycophenolate is of concern, as late renal flares can occur, and the influence of induction therapy on long-term renal outcomes might require follow-up for more than 5 years. ${ }^{25}$ It could take even longer to detect any influence on cardiovascular events. Furthermore, the dosage of mycophenolate is difficult to adjust in patients with renal insufficiency and requires monitoring. ${ }^{26}$

In view of the above caveats, and because patients with renal insufficiency (creatinine clearance $<30$ $\mathrm{ml} / \mathrm{min}$ and serum creatinine $>3 \mathrm{mg} / \mathrm{dl}[>265.2$ $\mu \mathrm{mol} / \mathrm{l}]$ in the Ginzler study) or other severe coexisting conditions were largely excluded from clinical studies, most nephrologists would probably use intravenous bolus dosing of cyclophosphamide and steroids in this subgroup of patients. Mycophenolate mofetil offers an alternative to cyclophosphamide for induction and maintenance therapy of patients with proliferative lupus nephritis

\section{Conclusion}

Mycophenolate mofetil is superior to intravenous cyclophosphamide for inducing renal remission, and has a significant advantage over cyclophosphamide for reducing ESRD or death. Furthermore, mycophenolate mofetil has lower risks of 
leukopenia, amenorrhoea and alopecia, but a higher risk of diarrhoea than cyclophosphamide. However, our conclusions need to be proved further in larger well designed trials.

Conflict of Interest: We have no conflict of interest.

\section{References}

1. Waldman M, Appel GB: Update on the treatment of lupus nephritis. Kidney Int 2006; 70: 14031412.

2. Font J, Ramos-Casals $\mathrm{M}$, Cervera $\mathrm{R}$ et al. Cardiovascular risk factors and the long-term outcome of lupus nephritis. QJM 2001; 94: 1926.

3. Dooley MA, Hogan S, Jennette $\mathrm{C}$ et al. Cyclophosphamide therapy for lupus nephritis: Poor renal survival in black Americans. Glomerular Disease Collaborative Network. Kidney Int 1997; 51: 1188- 1195.

4. Barr RG, Seliger S, Appel GB et al. Prognosis in proliferative lupus nephritis: The role of socio-economic status and race/ethnicity. Nephrol Dial Transplant 2003; 18: 2039- 2046.

5. Austin HA III, Klippel JH, Balow JE et al. Therapy of lupus nephritis: Controlled trial of prednisone and cytotoxic drugs. N Engl J Med 1986; 314 : 614- 619.

6. Steinberg AD, Decker JL: A double-blind controlled trial comparing cyclophosphamide, azathioprine and placebo in the treatment of lupus glomerulonephritis. Arthritis Rheum 1974; 17: 923- 937.

7. McCune WJ, Golbus J, Zeldes W et al. Clinical and immunologic effects of monthly administration of intravenous cyclophosphamide in severe systemic lupus erythematosus. N Engl J Med 1988; 318: 1423- 1431.

8. Ioannidis JP, Boki KA, Katsorida ME et al. Remission, relapse, and re-remission of proliferative lupus nephritis treated with cyclophosphamide. Kidney Int 2000; 57: 258264.
9. Petri M: Cyclophosphamide: New approaches for systemic lupus erythematosus. Lupus 2004; 13: 366-3671.

10. Kuiper-Geertsma DG, Derksen RH: Newer drugs for the treatment of lupus nephritis. Drugs 2003; 63: 167- 180.

11. Robson R, Cecka JM, Opelz G et al. Prospective registry-based observational cohort study of the long-term risk of malignancies in renal transplant patients treated with mycophenolate mofetil. Am J Transplant 2005; 5: 2954-2960.

12. Chan TM, Li FK, Tang CS et al. Efficacy of mycophenolate mofetil in patients with diffuse proliferative lupus nephritis. Hong KongGuangzhou Nephrology Study Group. N Engl J Med 2000; 343: 1156-1162.

13. Chan TM, Tse KC, Tang CS et al. Long-term study of mycophenolate mofetil as continuous induction and maintenance treatment for diffuse proliferative lupus nephritis. J Am Soc Nephrol 2005; 16: 1076- 1084.

14. Ong LM, Hooi LS, Lim TO et al. Randomized controlled trial of pulse intravenous cyclophosphamide versus mycophenolate mofetil in the induction therapy of proliferative lupus nephritis. Nephrology (Carlton) 2005; 10: 504-510.

15. $\mathrm{Hu} \mathrm{W}$, Liu Z, Chen $\mathrm{H}$ et al. Mycophenolate mofetil $v s$ cyclophosphamide therapy for patients with diffuse proliferative lupus nephritis. Chin Med J (Engl) 2002; 115: 705-709.

16. Ginzler EM, Dooley MA, Aranow C et al. Mycophenolate mofetil or intravenous cyclophosphamide for lupus nephritis. N Engl J Med 2005; 353: 2219- 2228.

17. Moore RA, Derry S: Systematic review and meta-analysis of randomised trials and cohort studies of mycophenolate mofetil in lupus nephritis. Arthritis Res Ther 2006; 8: R 182. 
18. Walsh M, James M, Jayne D et al. Mycophenolate mofetil for induction therapy of lupus nephritis: A systematic review and meta-analysis. Clin J Am Soc Nephrol 2007; 2: 968- 975.

19. Zhu B, Chen N, Lin Y et al. Mycophenolate mofetil in induction and maintenance therapy of severe lupus nephritis: A meta-analysis of randomized controlled trials. Nephrol Dial Transplant 2007; 22: 1933-1942.

20. G B Appel, G Contreras, M A Dooley et al. Mycophenolate Mofetil versus Cyclophosphamide for Induction Treatment of Lupus Nephritis. 2009. Published online ahead of print publication. www.jasn.org cited by 581 Related articles.

21.C Burchardi, D Scholondorff. Induction Therapy for Active Lupus Nephritis: Mycophenolate Mofetil versus Cyclophosphamide. Nat Clin Pract Nephrol. 2006; 2(6): 314-315.

22. Houssiau FA et al. Early response to immuno suppressive therapy predicts good renal outcome in lupus nephritis: lessons from long term follow up of patients in Euro-Lupus Nephritis Trial. Arthritis Rheum. 2004; 50: 3934-3940.
23. Contreras $G$ et al. Sequential therapies for proliferative lupus nephritis. N Engl J Med. 2004; 350: 970-980.

24. Chan TM et al. Long term study of mycophenolate mofetil as continuous induction and maintenance treatment for diffuse proliferative lupus nephritis. J Am Soc Nephrol. 2005; 16: 1076-1084.

25. Austin HA 3rd et al. Therapy of lupus nephritis: Controlled trial of prednisolone and cytotoxic drugs. N Engl J Med. 1986; 314: 614-619.

26. Shaw LM et al. Pharmacokinetics and concentration control investigations of mycophenolic acid in adults after transplantation. The Drug Monit. 2000; 22: 14-19. 